\title{
MSSB to Prevent Cable Termination Faults for Long High Voltage Underground Cable Lines
}

\author{
Bahadir Akbal \\ Department of Electric and Electronics Engineering, Konya Technical University, \\ Konya, Turkey \\ bakbal@ktun.edu.tr
}

\begin{abstract}
Cable termination fault is one of the most important problems for high voltage underground cable lines (HVUCL). Harmonic current (HC) and the sheath current (SC) are causes of cable termination faults of HVUCL. The sheath voltage (SV) of cable increases due to $\mathrm{SC}$ and $\mathrm{HC}$ also. So, the electroshock risk for human occurs. In this study, cable termination faults are examined for long HVUCL. In literature, solid bonding (SB) and sectional solid bonding (SSB) methods are used to reduce harmonic distortion rate and SC effect in HVUCL. However, when bonding of long HVUCL is made with SB and SSB, the limits of touch voltage and harmonic distortion are exceeded. Therefore, bonding of long HVUCL is made by the modified SSB (MSSB). In MSSB, SV of HVUCL should be known. Thus SV of HVUCL is forecasted by using artificial neural network and hybrid artificial neural networks (HANN) before the long HVUCL is installed. MSSB parameters and HANN are optimized by optimization method. The limits of touch voltage and harmonic distortion are restricted by using MSSB in long HVUCL under high harmonic distortion conditions.
\end{abstract}

Index Terms-Bonding method; High voltage cable termination; Hybrid artificial neural network; Insulation fault.

\section{INTRODUCTION}

High voltage underground cables (HVUC) are used in city centre or crowded areas because safety of the HVUC is better than of overhead lines. High voltage underground cable line (HVUCL) is used in submarine link also [1]. The fact that HVUC is used for various applications demonstrates that cable faults are very important problem for electric transmission, and cable faults are generally seen as cable termination faults.

connection between HVUC and high voltage equipment is is made by using cable terminations. Single core HVUC is shown in Fig. 1 [2], and cable terminations of single core HVUC are shown in Fig. 2(a) and Fig. 2(b). A cable termination fault of HVUC is shown in Fig. 3.

Electrical stress, increased voltage and thermal effects are the aging causes of insulation of HVUC [3], [4]. Cable termination voltage increases due to capacitive load current, proximity effect, high frequency, voltage transient, and parallel resonance [5]. Cable temperature increases due to overloading, harmonic current (HC), sheath current (SC), and environmental conditions [6].

Manuscript received 2 December, 2018; accepted 8 April, 2019.

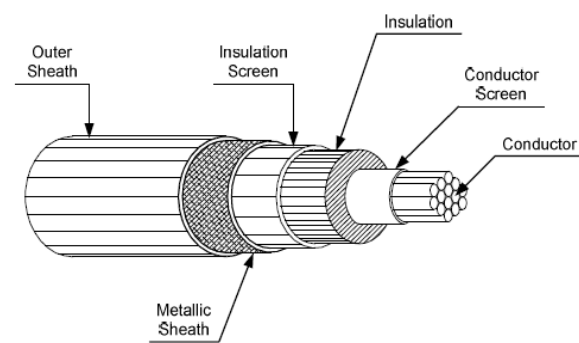

Fig. 1. High voltage underground cable.
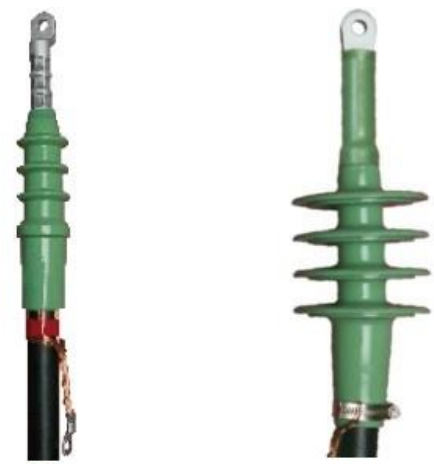

(a)

(b)

Fig. 2. a) Indoor cable termination; b) Outdoor cable termination.

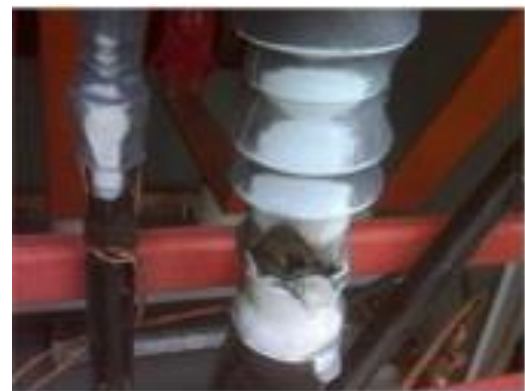

Fig. 3. High voltage underground cable termination fault.

$\mathrm{SC}$ is important factor for cable termination fault, and the most important factors for forming of SC are unbalanced phase current and harmonics. In three phase system, if three phase currents are not equal to each other, the balance of total magnetic field changes. Thus, SC and harmonic currents occur on the metallic sheath of high voltage cable. The effective factors for forming of SC are: HVUCL length $(L)$, distance of between phases $(d)$, and grounding resistance $(R g)$ [7]-[9].

Sheath voltage (SV) occurs due to $\mathrm{SC}$ and harmonic current, and SV is the cause of cable fault and electroshock 
for a human. SV of different HVUCL are examined in [10], but harmonic distortion rates of these lines are not considered.

SV effect is reduced by using bonding methods [11]-[16]. The bonding of HVUC is made according to IEEE 575-1988 standard. The bonding methods are: single point (SPB) bonding, solid bonding (SB), and cross bonding (CB). In these methods, if unbalanced phase current value is at a high value, SV exceeds touch voltage limit for a human. Thus, the optimized sectional solid bonding (OSSB) method is used to reduce SV for $1 \mathrm{~km}$ length HVUCL. High harmonic distortion is considered in this method [17], [18]. When OSSB is used, SV does not exceed touch voltage limit for human. However, if length of HVUCL exceeds $1 \mathrm{~km}$, harmonic distortion rate and SV increase, so that cable termination fault occurs in HVUCL.

In this study, various HVUCL are generated. These HVUCL are simulated in PSCAD/EMTDC simulation software. In the simulation studies, long HVUCL (length > $1 \mathrm{~km}$ ), that is going to be connected to electric network with high harmonic distortion rate, is considered to supply loads. The connection of the cable line is summarized in Fig. 4.

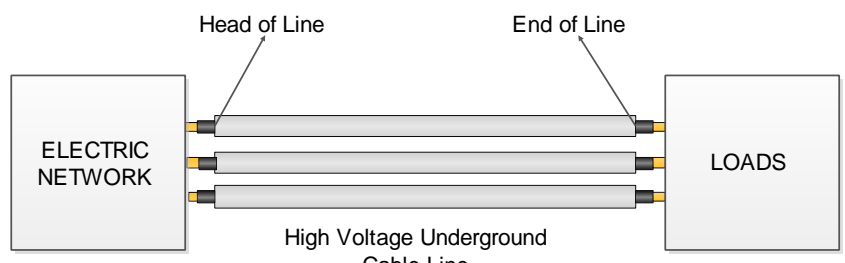

Fig. 4. High voltage underground cable termination fault.

\section{MATERIAL AND METHOD}

High voltage studies are generally made by using electromagnetic transient program [19]. In this study, PSCAD/EMTDC simulation program is used as electromagnetic transient program for simulation of long HVUCL. Single core HVUC cable (Fig. 5) is used for simulation studies. In literature, there are SPB, SB, and CB methods, and these methods do not prevent SC and SV effects in high harmonic case. Therefore, the SSB method, shown in Fig. 6, is developed to reduce SV effects [18]. However, SSB method is not sufficient to prevent SV effects in long HVUCL because the harmonic distortion rate and SV extremely increase on cable terminations of HVUCL. Thus, the modified SSB (MSSB) is developed, and MSSB method is shown in Fig. 7.

If length of HVUCL increases, its capacitance and harmonic distortion rate increases also due to the high capacitance value. Harmonics and capacitance of HVUCL are the causes of parallel resonance, so that SV of HVUCL exceeds the touch voltage limit. Thus, grounding inductance $(\mathrm{Lg})$ is used in MSSB method to prevent parallel resonance in HVUCL. In MSSB, the entire length of the line is called a "major part", and the major part consists of minor parts. The minor part parameters are minor part length $(L)$, distance of between phases $(d)$, grounding resistance $(R g)$, and grounding inductance $(L g)$. These parameters should be optimized to obtain the most economical and technical results. Values of minor part parameters should be determined before the long HVUCL is installed for using of MSSB.

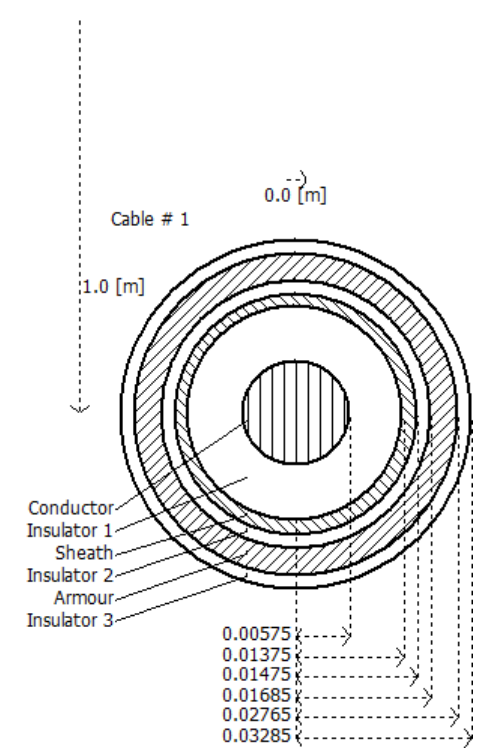

Fig. 5. The modeled single core high voltage underground cable in PSCAD.

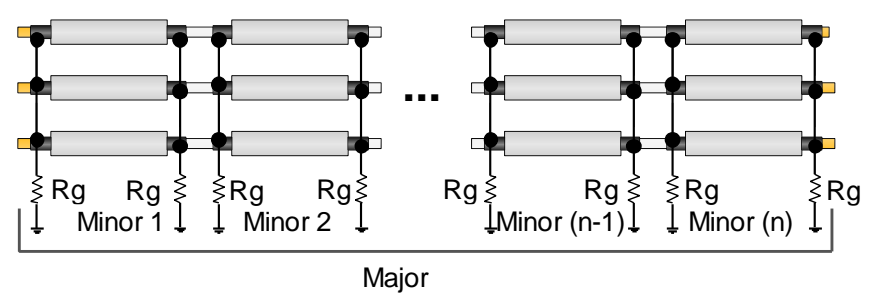

Fig. 6. The sectional solid bonding.

It is desired that MSSB parameters are determined before this line is installed to prevent the cable faults and electroshock. However, the SV of HVUCL is not known because this line is not installed yet. Thus SV of HVUCL is forecasted by using different artificial neural network (ANN) and hybrid artificial neural network (HANN). ANN is used in electric engineering to solve the forecasting problems. Mathematical model of human learning is used as working principle in ANN. ANN occurs from input layer, hidden layer, and output layer [20]-[22]. Also, neurons work as a transfer function in (1)

$$
y_{i}=f_{i}\left(\sum_{j=1}^{n} w_{i j} \times x_{j}+b_{i}\right),
$$

where $x_{j}$ is input, $w_{i j}$ is weight, $b_{i}$ is bias, $f_{i}$ is transfer function, and $\mathrm{y}_{\mathrm{i}}$ is output of the neuron.

The Mean square error (MSE) method that is shown in (2) is used to calculate training and forecasting errors

$$
E(t)=\frac{1}{n} \sum_{i=1}^{n}(p(i)-o(i))^{2},
$$

where $E(t)$ is forecasting error at $t^{\text {th }}$ iteration, $p(i)$ is the desired value for $i^{\text {th }}$ output, and o(i) is the real value for $i^{\text {th }}$ output. Weights of ANN must be updated to reduce the training error. 


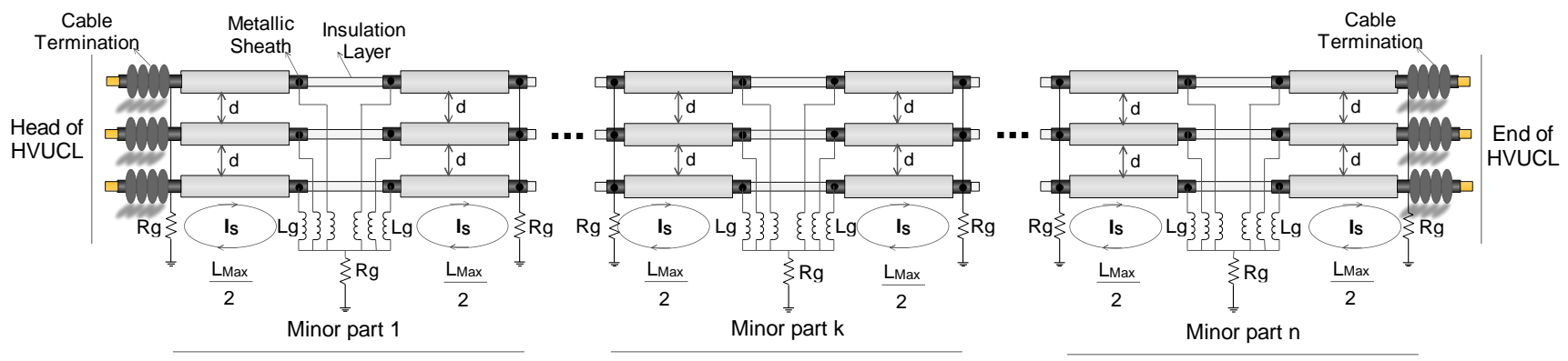

Fig. 7. Modified sectional solid bonding.

Major Part

Weights of classic ANN are updated by (3)

$$
w_{i}(t+1)=w_{i}(t)+\Delta w_{i}(t)
$$

In HANN method, weights of ANN are updated by optimization methods instead of (3). The flow chart of HANN method is shown in Fig. 8.

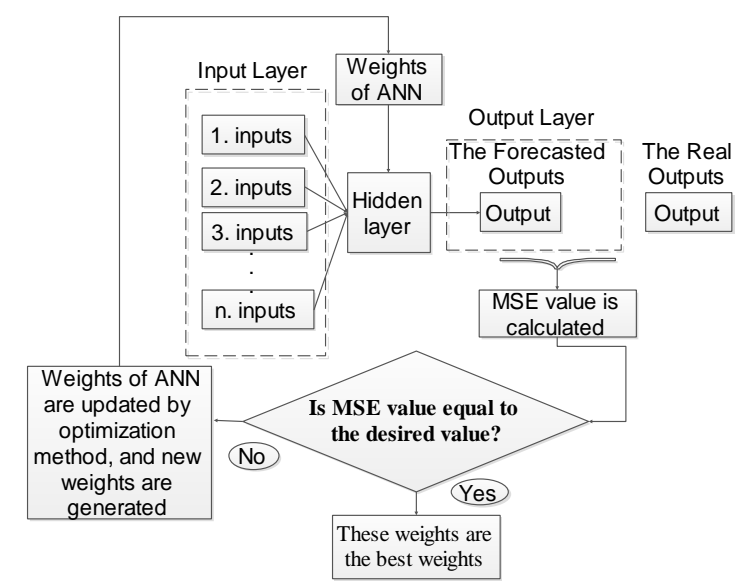

Fig. 8. Hybrid ANN flow chart.

Various HVUCL are simulated in PSCAD to obtain input and output (target) matrices for training and forecasting studies in ANN and HANN. Three phase currents $(I a, I b$, $I c)$, neutral current $(I n)$, harmonic currents $\left(I h_{1}, I h_{2}, I h_{3}\right)$, $T H D I, T H D V, L, d$, and $R g$ are effective on SV of end of line according to statistical analysis results. These 12 factors are the input parameters for the input matrix. The measured SV is the output parameter for the target matrix.

After training and forecasting errors of ANN and HANN methods are determined, the best method is selected among these methods for forecasting of SV. minor part parameters can be optimized after SV of HVUCL is estimated. $L, d$, and $R g$ are minor part parameters. If $L$ is maximum according to touch voltage limit, the minor part number of the major part is minimal. Thus minimum cost can be obtained in bonding of HVUCL. Also, if $d$ value is minimum, cable channel size becomes at minimum value. Therefore, values of $L_{M A X}, d_{M I N}$ and $R g$ for minor part are determined by optimization methods. The flow chart of optimisation of $L_{M A X}, d_{M I N}$, and $R g$ is shown in Fig. 9.

Vectors occur the input matrix, and 12 parameters that are $I a, I b, I c, I n, I h_{1}, I h_{2}, I h_{3}, T H D I, T H D V$, and $L_{M A X}, d_{M I N}$, and $R g$ occur a vector. SV occuts the output matrix (Fig. 9).

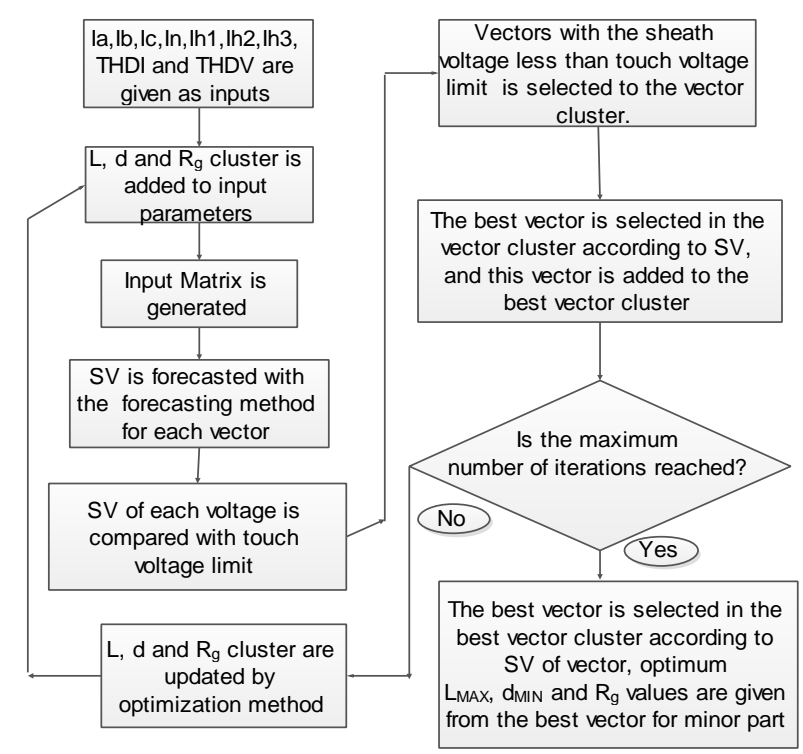

Fig. 9. Flow chart of optimisation of $L_{M A X}, d_{M I N}, R g$.

When HVUCL is simulated in PSCAD, $I a, I b, I c, I n, I h_{l}$, $I h_{2}, I h_{3}, T H D I$, and THDV values are kept constant, and $L_{M A X}, d_{M I N}$, and $R g$ values are changed. So, for the output matrix, different sheath voltages of HVUCL are measured according to these parameters in PSCAD. While a vector is generated for the input matrix, $L_{M A X}, d_{M I N}$, and $R g$ values are taken from $L, d$, and $R g$ cluster and added to $I a, I b, I c, I n$, $I h_{1}, I h_{2}, I h_{3}, T H D I$, and $T H D V$ parameters in ANN and HANN. This case is shown in Fig. 10. Thus, input matrix is generated by vectors, and SV are forecasted by using input matrix. The estimated sheath voltages are used as objective function value to determine $L_{M A X}, d_{M I N}$ and $R g$ values.

Optimum $L g$ is determined according to optimum $L_{M A X}$, $d_{M I N}$, and $R g$ values. The flow chart that is used to determine optimum $L g$ is shown in Fig. 11.

In this case, the determined optimum $L_{M a x}, d_{M \text { Min }}$, and $R g$ for minor part are kept constant, and $L g$ value is changed, and difference of harmonic distortion rates between head and end of cable terminations is measured according to different $L g$ values in PSCAD. So, input and output matrices are occurred for training and forecasting process of the forecasting method for optimization of $L g$. The difference of harmonic distortion rates between head and end of cable terminations is used as the fitness value for optimization of $L g$ also. The difference of harmonic distortion rates between head of HVUCL and end of HVUCL should be minimum value to determine optimum $L g$. 


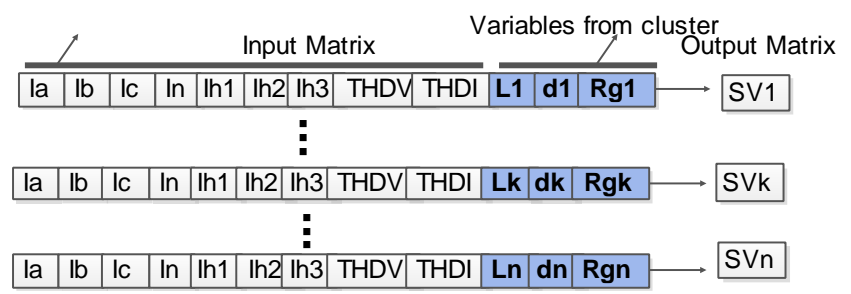

Fig. 10. Vector cluster and outputs for optimization of LMAX, dMIN, and Rg.

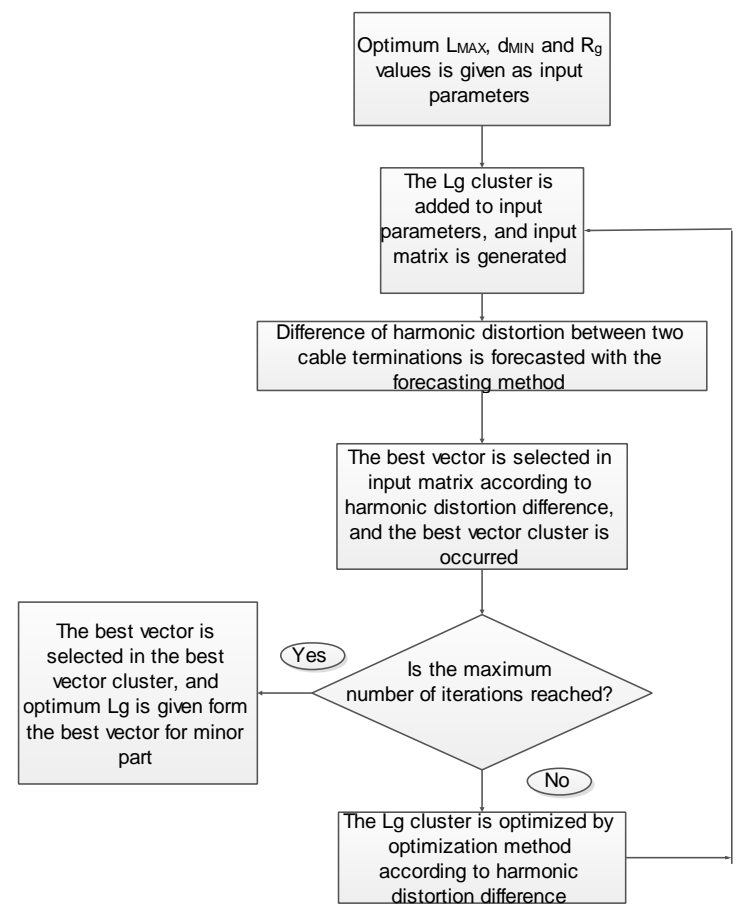

Fig. 11. Flow chart of optimisation of Lg.

In $L g$ optimisation, vectors occur the input matrix, and difference of harmonic distortion rates between two cable terminations occurs the output (target) matrix. When a vector is generated, $L g$ value is taken from the $L g$ cluster and is added to $L_{M a x}, d_{M I i n}$, and $R g$ parameters. This case is shown in Fig. 12. So, a vector is occurred and the input matrix is generated from the generated vectors.

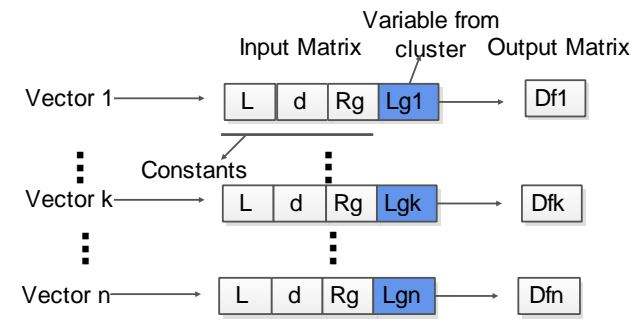

Fig. 12. Vector cluster and outputs for optimization of $\mathrm{Lg}$.

\section{RESULTS AND DISCUSSIONS}

Primarily, $L_{\text {Max }}, d_{\text {Min }}$, and $R g$ should be determined before MSSB method is applied to HVUCL. In this study, 287 various HVUCL are simulated in PSCAD. The simulation results of these HVUCL are used for training and forecasting studies of ANN and HANN. There are used 12 parameters to input parameters. These parameters are $I a, I b, I c, I n, I h_{1}, I h_{2}$, $I h_{3}, T H D I$, and $T H D V$. The sheath voltages of these lines are measured in PSCAD at the end of simulation studies, and the measured sheath voltages are used for the output matrix. Thus, $287 \times 12$ data matrix is used as the input matrix, while
$287 \times 1$ data matrix is used as the output matrix for training of ANN and HANN. Also, $31 \times 12$ data matrix is used as the input matrix, and $31 \times 1$ data matrix is used as the output for forecasting of ANN and HANN. The training and forecasting errors of ANN and HANN methods are shown in Table I. Forecasting errors of HANN methods are lower than of ANN methods. Therefore, HANN methods are used for forecasting studies. Differential evolution algorithm (DEA), genetic algorithm (GA), and particle swarm optimization (PSO) are used as optimization methods. ANN is also optimized by using these optimization methods. HANN methods are developed as PSO-ANN, DEA-ANN, and GAANN. The flow chart that is shown in Fig. 8 is used for hybrid ANN methods. Errors of training and forecasting of the forecasting methods are shown in Table I. $L_{M a x}, d_{M i n}$, and $R g$ are determined by optimization methods for minor parts. The determined $\mathrm{L}_{\mathrm{Max}}, d_{M i n}$, and $R g$ values are shown in Table II. While $L_{M a x}, d_{M i n}$, and $R g$ values are determined, optimization method that is used for optimization of HANN is used for updating of $L, d$ and $R g$ cluster. For example, if HANN is optimized by GA for GA-ANN, GA is used to update $L, d$, and $R g$ cluster.

TABLE I. TRAINING AND FORECASTING ERRORS OF DIFFERENT TYPE ANN MODELS.

\begin{tabular}{|c|c|c|}
\hline ANN type & $\begin{array}{c}\text { Training } \\
\text { Errors }\end{array}$ & $\begin{array}{c}\text { Forecasting } \\
\text { Errors }\end{array}$ \\
\hline Feedforward backpropagation & 0.0034 & 2.9642 \\
\hline Layer recurrent & 0.0763 & 3.3381 \\
\hline Linear Layer & 18.6157 & 171.1096 \\
\hline NARX & 0.1384 & 3.5480 \\
\hline Perceptron & 12.6991 & 116.9519 \\
\hline PSO-ANN & 0.1213 & 0.4452 \\
\hline DEA-ANN & 0.1286 & 0.3869 \\
\hline GA-ANN & 0.1180 & 0.4273 \\
\hline
\end{tabular}

After $L_{M a x}, d_{M i n}$, and $R g$ values are determined, the major part is divided into minor parts according to $L_{\operatorname{Max}}$ of the minor part. While MSSB is used for bonding of long HVUCL, $L_{M a x}, d_{M i n}$, and $R g$ values are kept as constant and $L g$ values are changed. So, 143 bonded different HVUCL are simulated. The difference of harmonic distortion rates between cable terminations is measured in PSCAD. Optimum $L g$ value is determined by optimization methods and HANN methods.

TABLE II. THE DETERMINED OPTIMUM MINOR PART PARAMETERS.

\begin{tabular}{|c|c|c|c|}
\hline $\begin{array}{c}\text { Optimization } \\
\text { Method }\end{array}$ & $\boldsymbol{L}_{\text {Max }}(\boldsymbol{m})$ & $\boldsymbol{d}_{\boldsymbol{M i n}}(\boldsymbol{m})$ & $\boldsymbol{R g}(\boldsymbol{o h m})$ \\
\hline PSO & 387 & 0.3091 & 22.30 \\
\hline DEA & 389 & 0.4681 & 33.15 \\
\hline GA & 375 & 0.3319 & 13.27 \\
\hline
\end{tabular}

Similarly, while optimum $L g$ value is determined, optimization method that is used for optimization of HANN is used for updating of $L g$ cluster also. $143 \times 3$ matrix is used as the input matrix and the $143 \times 1$ matrix is used as the output matrix for the training of HANN. $25 \times 3$ matrix is used as the input matrix and $25 \times 1$ matrix is used as the output matrix for the forecasting of HANN. The results of HANN and optimization methods are shown in Table III. 
TABLE III. TRAINING AND FORECASTING ERRORS OF HANN AND THE DETERMINED OPTIMUM LG VALUES.

\begin{tabular}{|c|c|c|c|c|}
\hline $\begin{array}{c}\text { Optimization } \\
\text { Method }\end{array}$ & $\begin{array}{c}\text { HANN } \\
\text { Method }\end{array}$ & $\begin{array}{c}\text { Training } \\
\text { Error }\end{array}$ & $\begin{array}{c}\text { Forecasting } \\
\text { Error }\end{array}$ & $\begin{array}{c}\text { Optimum } \\
\text { Lg Value } \\
\text { (Henry) }\end{array}$ \\
\hline PSO & PSO-ANN & 0.0775 & 0.1200 & 0.0636 \\
\hline DEA & DEA-ANN & 0.1286 & 0.3869 & 0.0422 \\
\hline GA & GA-ANN & 0.0602 & 0.2044 & 0.0309 \\
\hline
\end{tabular}

After the optimum $L_{\text {Max }}, d_{\text {Min }}, R g$, and $L g$ parameters are determined for the minor part, these parameters are used in MSSB for bonding of HVUCL, and HVUCL is simulated in PSCAD.

In simulation studies, total voltage harmonic distortion of the electric network is determined as $5 \%$ according to IEEE STD 519-1992 Harmonic Limits, and touch voltage limit is $71 \mathrm{~V}$ peak (50 V rms) according to IEC 479-1 standard.

$I a, I b, I c, I n, I h_{l}, I h_{2}$, and $I h_{3}$ parameter values are 14.17 A, 442.84 A, 14.17 A, 422.37 A 23.90 A, 28.29 A, and $28.11 \mathrm{~A}$, respectively. The selected length for long HVUCL is $5 \mathrm{~km}$.

If bonding of HVUCL is made by solid bonding method under these conditions, harmonic distortion rate and SV values exceed limits (Fig. 13). Thus, cable termination faults and electroshock can occur in long HVUCL.

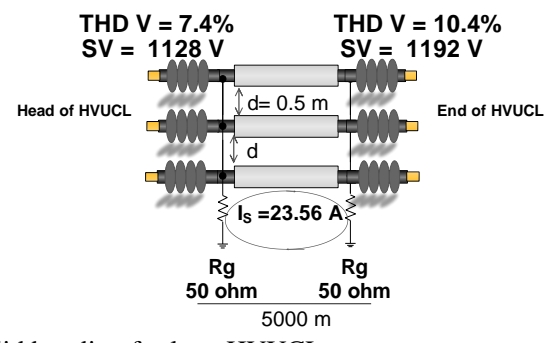

Fig. 13. Solid bonding for long HVUCL.

SSB is used to solve SV problems in literature also. When SSB is optimized by GA, PSO, and DEA, the simulation results of SSB are shown in Fig. 14, Fig. 15, and Fig. 16, respectively. In simulation results of SSB methods, harmonic distortion rates of cable terminations exceed $5 \%$. So, cable termination faults can occur in long HVUCL. Also, the sheath voltages of cable terminations exceed touch voltage limit, so electroshock can occur in long HVUCL. Namely, solid bonding and SSB are not suitable for bonding of long HVUCL under these conditions.

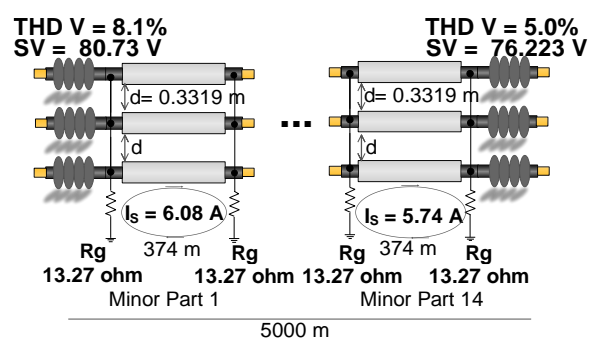

Fig. 14. Simulation result of SSB that is optimized with GA.

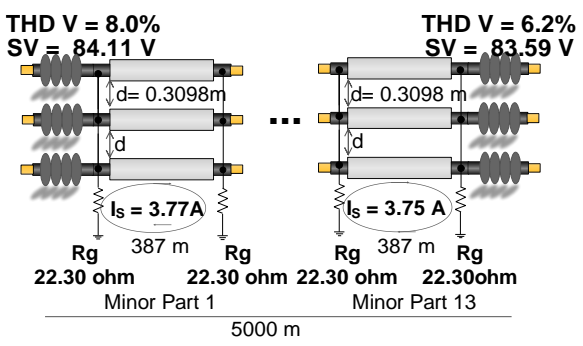

Fig. 15. Simulation result of SSB that is optimized with PSO.

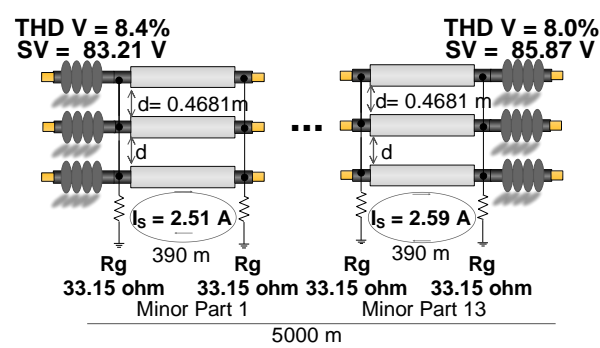

Fig. 16. Simulation result of SSB that is optimized with DEA.

When MSSB method is optimized by GA, PSO, and DEA, the simulation results of MSSB are shown in Fig. 17, Fig. 18, and Fig. 19, respectively.

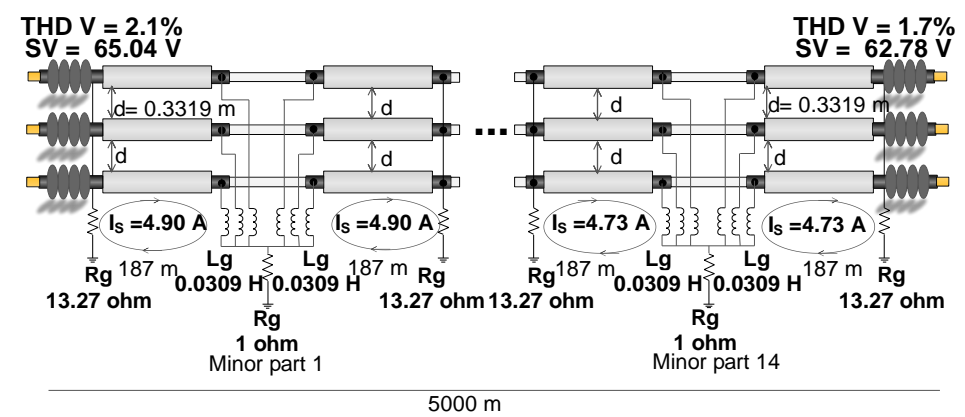

Fig. 17. Simulation result of MSSB that is optimized with GA.

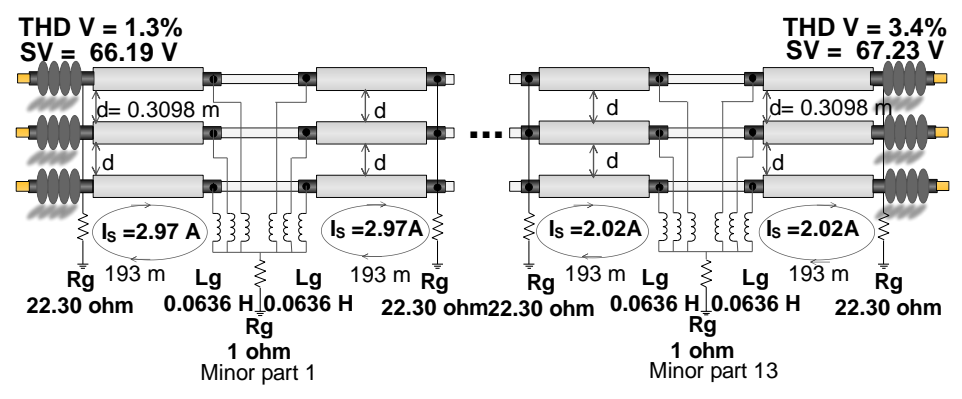

Fig. 18. Simulation result of MSSB that is optimized with PSO. 


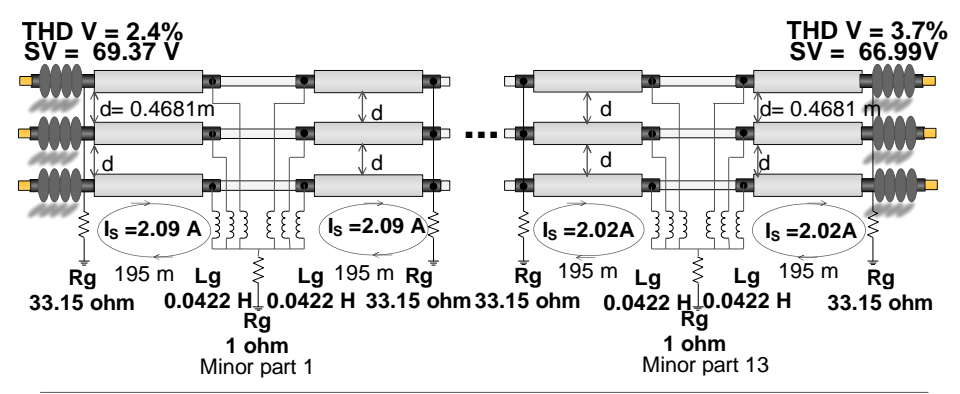

Fig. 19. Simulation result of MSSB that is optimized with DEA.

Harmonic distortion rates of MSSB methods do not exceed $5 \%$, and the sheath voltages of MSSB methods do not exceed touch voltage limit. When MSSB is optimized with GA, the difference of harmonic distortion rates between head and end of cable termination is at a very low value.

\section{CONCLUSIONS}

The MSSB method is improved for bonding of long HVUCL to avoid cable faults, which is connected to the electric network with high harmonic distortion rate. However, MSSB parameters should be determined before this HVUCL is installed at the project phase, and SV of HVUCL should be known to determine the MSSB parameters.

Thus, simulation of various HVUCL are made in PSCAD/EMTDC and the simulation results are used for the forecasting of SV of HVUCL. Different ANN network types and HANN are used to estimate SV of long HVUCL that will be installed as a new line. HANN methods are DEAANN, PSO-ANN, and GA-ANN. The forecasting errors of HANN methods are less than ANN methods. Also, the forecasting error of DEA-ANN is 0.3869 , and this value is the smallest forecasting error among the HANN methods. After SV of HVUCL is estimated by HANN methods, MSSB parameters are determined. In literature, SB and SSB methods are used as bonding methods also. When bonding of long HVUCL is made by SB and SSB methods, the harmonic distortions of these methods exceed harmonic distortion limit, and SV of these methods exceed the touch voltage limit. When bonding of long HVUCL is made by MSSB, the harmonic distortion and SV of MSSB does not exceed the limits. Particularly, when optimization of MSSB and HANN is made by GA, cable terminations voltages are measured as $65.04 \mathrm{~V}$ and $62.78 \mathrm{~V}$. Harmonic distortions are measured as $2.1 \%$ and $1.7 \%$. These values are under the determined limit values.

\section{CONFLICTS OF INTEREST}

The authors declare that they have no conflicts of interest.

\section{REFERENCES}

[1] R. Benato, I. Balanuye, F. Köksal, N. Ozan, and E. Özdemirci, “A 4 GW AC submarine Turkish power grid reinforcement under the Dardanelles Strait", in Proc. of IEEE 2017 AEIT International Annual Conference, Cagliari, Italy, pp. 1-6, 2017. DOI: 10.23919/AEIT.2017.8240507.

[2] A. D. Tziouvaras, "Protection of high-voltage AC cables", in Proc. of $59^{\text {th }}$ Annual Conf. for Protective Relay Engineers, TX, USA, pp. 4861, 2006. DOI: 10.1109/CPRE.2006.1638691.
[3] B. Yunus, "Trend adjusted lifetime monitoring of underground power cable", Electric Power Systems Research, vol. 143, pp. 189-196, 2017. DOI: 10.1016/j.epsr.2016.10.045.

[4] L. Bessissa, L. Boukezzi, and D. Mahi, "Influence of fuzzy parameters on the modeling quality of XLPE insulation properties under thermal aging", Fuzzy Information and Engineering, vol. 8, no. 1, pp. 101-112, 2016. DOI: 10.1016/j.fiae.2016.03.006.

[5] C. L. Bak and F. F. Silva, "High voltage AC underground cable systems for power transmission - A review of the Danish experience, part 1", Electric Power Systems Research, vol. 140, pp. 984-994, 2016. DOI: 10.1016/j.epsr.2016.05.034.

[6] C. L. Bak and F. F. Silva, "High voltage AC underground cable systems for power transmission - A review of the Danish experience: part 2", Electric Power Systems Research, vol. 140, pp. 995-1004, 2016. DOI: $10.1016 /$ j.epsr.2016.05.035.

[7] W. Moutassem and G. J. Anders, "Calculation of the eddy current and hysteresis losses in sheathed cables inside a steel pipe", IEEE Trans. Power Delivery, vol. 25, pp. 2054-2063, 2010. DOI: 10.1109/TPWRD.2010.2049509.

[8] B. Akbal, "Neural-network-based current forecasting on high-voltage underground cables", Electronics World, vol. 122, no. 1959, pp. 3034, 2016.

[9] R. Benato, "Multiconductor analysis of underground power transmission systems: EHV AC cables", Electric Power Systems Research, vol. 79, no. 1, pp. 27-38, 2009. DOI: 10.1016/j.epsr.2008.05.016.

[10] M. Shaban, M. A. Salam, S. P. Ang, and W. Voon, "Induced sheath voltage in power cables: A review", Renewable and Sustainable Energy Reviews, vol. 62, pp. 1236-1251, 2016. DOI: 10.1016/j.rser.2016.05.032.

[11] J. R. Ruiz, A. Garcia, and X. A. Morera, "Circulating sheath currents in flat formation underground power lines", in Proc. of 2007 International Conf. Renewable Energies and Power Quality, pp. 15, 2007. DOI: 10.24084/repqj05.217.

[12] L. Zhonglei, B. X. Du, L. Wang, C. Yang, and H. J. Liu, "The calculation of circulating current for the single-core cables in smart grid", in Proc. of 2012 IEEE Innovative Smart Grid Technologies Asia, pp. 1-4, 2012. DOI: 10.1109/ISGT-Asia.2012.6303176.

[13] S. Czapp, K. Dobrzynski, J. Klucznik, and Z. Lubosny, "Calculation of induced sheath voltages in power cables - Single circuit system versus double circuit system", Journal of Information, Control and Management Systems, vol. 12, pp. 113-123, 2014.

[14] C. K. Jung, J. B. Lee, and J. W. Kang, "Sheath circulating current analysis of a cross-bonded power cable systems", J. Electr. Eng. \& Technol., vol. 2, pp. 320-328, 2007. DOI: 10.5370/JEET.2007.2.3.320.

[15] K. V. Gouramanis, C. G. Kaloudas, T. A. Papadopoulos, G. K. Papagiannis, and E. K. Stasinos, "Sheath voltage calculations in long medium voltage power cables", in Proc. of IEEE Trondheim Power Tech, Norway, pp. 1-7, 2011. DOI: 10.1109/PTC.2011.6019234.

[16] C. K. Jung, J. B. Lee, J. W. Kang, X. H. Wang, and Y. H. Song, "Sheath current characteristic and its reduction on underground power cable systems", in Proc. of IEEE Power Engineering Society General Meeting, CA, USA, pp. 2562-2569, 2005. DOI: 10.1109/PES.2005.1489525.

[17] B. Akbal, "Sectional solid bonding for grounding of high voltage underground cables to reduce the sheath current effects", International Journal of Innovative Research in Engineering \& Management, vol. 2, no. 2, pp. 103-107, 2016.

[18] B. Akbal, "OSSB and hybrid methods to prevent cable faults for harmonic containing networks", Elektronika ir Elektrotechnika, vol. 24, no. 2, pp. 31-36, 2018. DOI: 10.5755/j01.eie.24.2.20633.

[19] U. S. Gudmundsdottir, B. Gustavsen, C. L. Bak, and W. Wiechowski, 
"Field test and simulation of a 400-kV cross-bonded cable system", IEEE Transactions on Power Delivery, vol. 26, pp. 1403-1410, 2011. DOI: 10.1109/TPWRD.2010.2084600.

[20] R. Achanta, "Long term electric load forecasting using neural networks and support vector machines", International Journal of Computer Science and Technology, vol. 3, pp. 266-269, 2012. DOI: 10.1016/j.apm.2008.07.010.
[21] T. Weigerta, Q. Tianb, and Q. Lianb, "State-of-charge prediction of batteries and battery-supercapacitor hybrids using artificial neural networks", Journal of Power Sources, vol. 196, pp. 4061-4066, 2011. DOI: $10.1016 /$ j.jpowsour. 2010.10.075.

[22] W. Charytoniuk and M. S. Chen, "Very short-term load forecasting using artificial neural networks", IEEE Transactions on Power Systems, vol. 15, pp. 263-268, 2000. DOI: 10.1109/59.852131. 